\title{
Osteoclast Fusion and Fission
}

\author{
Ineke D. C. Jansen · Jenny A. F. Vermeer • \\ Veerle Bloemen · Jan Stap • Vincent Everts
}

Received: 14 July 2011 / Accepted: 1 April 2012/Published online: 25 April 2012

(C) The Author(s) 2012. This article is published with open access at Springerlink.com

\begin{abstract}
Osteoclasts are specialized multinucleated cells with the unique capacity to resorb bone. Despite insight into the various steps of the interaction of osteoclast precursors leading to osteoclast formation, surprisingly little is known about what happens with the multinucleated cell itself after it has been formed. Is fusion limited to the short period of its formation, or do osteoclasts have the capacity
\end{abstract}

The authors have stated that they have no conflict of interest.

Electronic supplementary material The online version of this article (doi:10.1007/s00223-012-9600-y) contains supplementary material, which is available to authorized users.

I. D. C. Jansen · J. A. F. Vermeer · V. Bloemen · V. Everts Department of Oral Cell Biology, Academic Centre for Dentistry Amsterdam (ACTA), Research Institute MOVE, University of Amsterdam and VU University Amsterdam, Gustav Mahlerlaan 3004, 1081 LA Amsterdam, The Netherlands

J. A. F. Vermeer

e-mail: j.vermeer@acta.nl

V. Bloemen

e-mail: veerle.bloemen@groept.be

V. Everts

e-mail: v.everts@acta.nl

I. D. C. Jansen $(\bowtie)$

Department of Periodontology, Academic Centre for Dentistry Amsterdam (ACTA), Research Institute MOVE, University of Amsterdam and VU University Amsterdam, Gustav Mahlerlaan 3004, 1081 LA Amsterdam, The Netherlands

e-mail: ineke.jansen@acta.nl

J. Stap

Van Leeuwenhoek Centre for Advanced Microscopy

(LCAM)-AMC, Department of Cell Biology and Histology,

Academic Medical Centre (AMC), University of Amsterdam,

Meibergdreef 15, 1005 AZ Amsterdam, The Netherlands

e-mail: j.stap@amc.uva.nl to change their size and number of nuclei at a later stage? To visualize these processes we analyzed osteoclasts generated in vitro with M-CSF and RANKL from mouse bone marrow and native osteoclasts isolated from rabbit bones by live cell microscopy. We show that osteoclasts fuse not only with mononuclear cells but also with other multinucleated cells. The most intriguing finding was fission of the osteoclasts. Osteoclasts were shown to have the capacity to generate functional multinucleated compartments as well as compartments that contained apoptotic nuclei. These compartments were separated from each other, each giving rise to a novel functional osteoclast or to a compartment that contained apoptotic nuclei. Our findings suggest that osteoclasts have the capacity to regulate their own population in number and function, probably to adapt quickly to changing situations.

Keywords Osteoclast - Bone marrow cell - Cell growth · Senescence $\cdot$ Apoptosis

Osteoclasts are multinucleated, polarized cells, with a unique function: resorption of mineralized substrates such as bone, dentin, and mineralized cartilage. They originate from mononuclear hematopoietic cells of the monocyte lineage. Supported by osteoblasts and bone lining cells, these mononuclear cells fuse and form multinucleated, tartrate-resistant acid phosphatase (TRAP)-positive, polarized cells [1]. The process of differentiation and fusion is modulated by the cytokines M-CSF and RANKL, which are expressed in vivo by osteoblast-like cells.

The formation of multinucleated bone resorbing osteoclasts is a multistep process comprising (1) recruitment of mononuclear precursors from the bone marrow or peripheral blood, (2) attraction of these cells by bone lining cells 
to the bone site where resorption is needed, (3) attachment of the precursors to the bone lining cells [2], (4) a subsequent differentiation of the attached precursors into mononuclear TRAP-positive cells, (5) migration of these osteoclast precursors to the mineralized surface, and finally (6) fusion and the formation of multinucleated osteoclasts.

Cell-cell interaction between the osteoblast-like bone lining cells and osteoclast precursors is crucial in these processes, and it has been shown that this interaction significantly alters gene expression and highly promotes the formation of osteoclasts [2, 3]. Zambonin et al. [4] showed already in 1984 with live cell imaging that monocytes fuse with osteoclasts and that these cells actively migrated to and from each other prior to the actual fusion, in this way allowing contact by continuous formation and retraction of lamellipodia and filopodia. Despite insight into the various steps of osteoclast precursor and osteoclast interaction, surprisingly little is known about what happens with the multinucleated cell itself after it has been formed. Is fusion limited to the short period of its formation, or do osteoclasts have the capacity to change their size and number of nuclei at a later stage, thus responding to new situations in bone degradation during their life span? Is it possible that, in addition to fusion of mononuclear cells with multinucleated ones, multinucleated cells fuse with each other? Is the alternative that multinucleated osteoclasts split up in more than one different multinucleated cell even possible? To gain insight into these different possibilities, we made use of a live cell imaging approach and visualized the interaction of osteoclast precursors and mature osteoclasts during a period of several days.

\section{Materials and Methods}

Mouse Bone Marrow Cell Culture with RANKL and M-CSF for the Generation of Osteoclasts

Osteoclasts were generated as described earlier by de Vries et al. [5]. Briefly, 6-week-old C57BL/6J mice were killed following a lethal peritoneal injection of sodium pentobarbital. Tibiae were dissected, cleaned of soft tissue, and ground in a mortar with alpha-minimal essential medium $(\alpha$-MEM; Invitrogen, Paisley, UK) supplemented with $5 \%$ fetal calf serum (FCS; HyClone, Logan, UT), $100 \mathrm{U} / \mathrm{mL}$ penicillin, $100 \mu \mathrm{g} / \mathrm{mL}$ streptomycin, $250 \mathrm{ng} / \mathrm{mL}$ amphotericin B (antibiotic antimycotic solution; Sigma, St. Louis, MO), and heparin (170 IE/mL). The cell suspension was aspirated through a 21-gauge needle and filtered over a 70 $\mu \mathrm{m}$-pore size Cell Strainer filter (Falcon/Becton Dickinson, Franklin Lakes, NJ). Cells were washed in culture medium, centrifuged ( $5 \mathrm{~min}, 200 \times g)$, and plated $\left(1.6 \times 10^{6}\right.$ cells/ $\mathrm{mL}$ ) in two-well, glass-bottomed chamber slides (Lab-Tek
II; Nunc, Roskilde, Denmark) with $1 \mathrm{~mL}$ culture medium containing $30 \mathrm{ng} / \mathrm{mL}$ recombinant murine M-CSF (R\&D Systems, Minneapolis, MN) and $20 \mathrm{ng} / \mathrm{mL}$ recombinant murine RANKL (R\&D Systems), 5 \% FCS, and antibiotics. Chamber slides were coated with carbon to promote cell attachment and spreading [6]. In addition, cells $\left(1.3 \times 10^{5} / \mathrm{mL}\right)$ were seeded on bovine cortical bone slices with a thickness of $650 \mu \mathrm{m}$.

Culture media were refreshed on the third day, and cells were cultured for another $68 \mathrm{~h}$ while they were simultaneously followed by live cell imaging.

\section{Native Osteoclasts}

Native osteoclasts were isolated from 5-day-old New Zealand white rabbits. Calvariae and long bones (tibiae) were dissected and collected in $10 \mathrm{~mL} \alpha$-MEM, with $1 \%$ antibiotics but without FCS. Bones were cut into very small fragments, and this homogenate was transferred to a $50-\mathrm{mL}$ tube in $35 \mathrm{~mL} \alpha$-MEM without FCS and with $1 \%$ antibiotic antimycotic solution. Fragments were gently shaken for $30 \mathrm{~s}$ to release the osteoclasts from the bone. After $90 \mathrm{~s}$ of sedimentation, the supernatant was collected. The last part of the procedure was repeated once more with $25 \mathrm{~mL}$ of $\alpha$-MEM. Supernatants were collected and centrifuged for $2 \mathrm{~min}$ at ambient temperature at $700 \mathrm{rpm}$. The pellet containing the osteoclasts was washed once with $50 \mathrm{~mL}$ $\alpha$-MEM containing $5 \%$ FCS, subsequently centrifuged, collected in $10 \mathrm{~mL}$ of $\alpha$-MEM containing $5 \%$ FCS and $1 \%$ antibiotics, and finally seeded in $25-\mathrm{cm}^{2}$ Costar (Corning, Corning, NY) culture flasks. After $48 \mathrm{~h}$ at $37^{\circ} \mathrm{C}$ in an atmosphere containing $5 \% \mathrm{CO}_{2}$, osteoclasts were monitored for $80 \mathrm{~h}$ by time lapse microscopy as described below.

\section{Time Lapse Microscopy and Image Processing}

Cells were imaged using a Leica IR-BE (Leica Microsystems, Wetzlar, Germany) inverted wide-field microscope at $37^{\circ} \mathrm{C}$ in an atmosphere containing $5 \% \mathrm{CO}_{2}$ [7]. Phase contrast images were acquired at 5- or 10-min time intervals using a $\times 40$ objective. Multifield imaging allowed simultaneous monitoring of different sites in one flask or well. Images were processed and analyzed using custom-made software and Image Pro Plus (Mediacybernetics, Carlsbad, CA).

Immunolocalization of ERMP12, ERMP20, F4/80, Moma2, ICAM1, and MMP9 in Osteoclastogenesis Cultures

Osteoclastogenesis cultures were performed as mentioned above, fixed after 3 and 4 days of culture with $4 \%$ PBS buffered formaldehyde, and subsequently washed with PBS. Before incubation with the primary antibodies, 
nonspecific binding was blocked with "image it Fx signal enhancer" (Invitrogen/Molecular Probes, Carlsbad, CA) for $30 \mathrm{~min}$ at ambient temperature. Primary antibodies were anti-MMP9 (goat anti-mouse MMP9 [R \& D Systems], used in a 1:100 dilution in PBS), anti-ICAM1 (rat antimouse ICAM1 [R \& D Systems], 1:100 diluted in PBS), anti-ERMP12 (CD31), anti-ERMP20 (Ly-6C), anti-Moma2, and anti-F4/80 (the last four were all rat antimouse and a gift of P. Leenen, Erasmus University, Rotterdam, the Netherlands; these antibodies were used in a 1:20 dilution in PBS). Incubation was at $4{ }^{\circ} \mathrm{C}$ overnight and subsequently for $1 \mathrm{~h}$ in ambient conditions; they were then washed two times with PBS and subsequently incubated for $2 \mathrm{~h}$ with a goat anti-rat Alexa 488 (Invitrogen, for MMP9) or goat anti-mouse Alexa-488 (for ER-MP12/20, Moma2, F4/80, ICAM1). Nuclei were visualized with DAPI staining (1.5 $\mu \mathrm{g} / \mathrm{mL}$ DAPI for $10 \mathrm{~min}$ ). After intensive washing, the procedure was finished by adding a drop of Vectashield (Vector Laboratories, Burlingame, CA) to enhance the fluorescence. Staining was visualized by a Leica IMDR converted fluorescence microscope equipped with a digital camera (Leica DFC 320).

\section{Actin and CD44 Staining of Osteoclasts Generated from Mouse Bone Marrow}

Mouse bone marrow cells were seeded on cortical bone slices, and osteoclastogenesis was induced during a culture period of 8 days in the presence of M-CSF and RANKL, as described above. Osteoclast plasma membranes were visualized by staining these with anti-CD44 as described previously [5]. In short, bone slices were washed in PBS, fixed in $4 \%$ PBS buffered formaldehyde for $5 \mathrm{~min}$, and subsequently washed in PBS. Nonspecific binding to cells was blocked for 30 min with $10 \%$ normal goat serum (Vector Laboratories), followed by overnight incubation at $4{ }^{\circ} \mathrm{C}$ with rat anti-mouse CD44 antibody IM7.8.1 1:200 in PBS/1 \% BSA (Cedarlane Laboratories, Burlington, Canada). Subsequently, slices were washed three times with PBS and incubated for 60 min with the secondary goat-antirat Alexa 647-conjugated antibody (Invitrogen). Following three PBS washes, F-actin was stained as described previously [8] using Alexa 488-phalloidin (Invitrogen). Finally, nuclei were stained with propidium iodide (Sigma). Image stacks were generated with a confocal laser scanning microscope (Leica) using an argon laser (for Alexa 488 and propidium iodide) and a helium laser (for Alexa 647).

\section{Results}

Formation of Multinucleated Cells by Fusion

Bone marrow cells isolated from mouse tibiae were cultured on plastic in the presence of M-CSF and RANKL and monitored after 3 days of culture by live cell imaging for a subsequent 68 h. Frequently, fusion was noted between mononuclear cells but also between two multinucleated cells and between a mononuclear cell and a multinucleated cell. Prior to fusion, cells migrated to each other and subsequently made contact as if to find an appropriate site for fusion. They interacted with each other by membrane extensions. These interactions were characterized by a relatively short moment of contact with the plasma
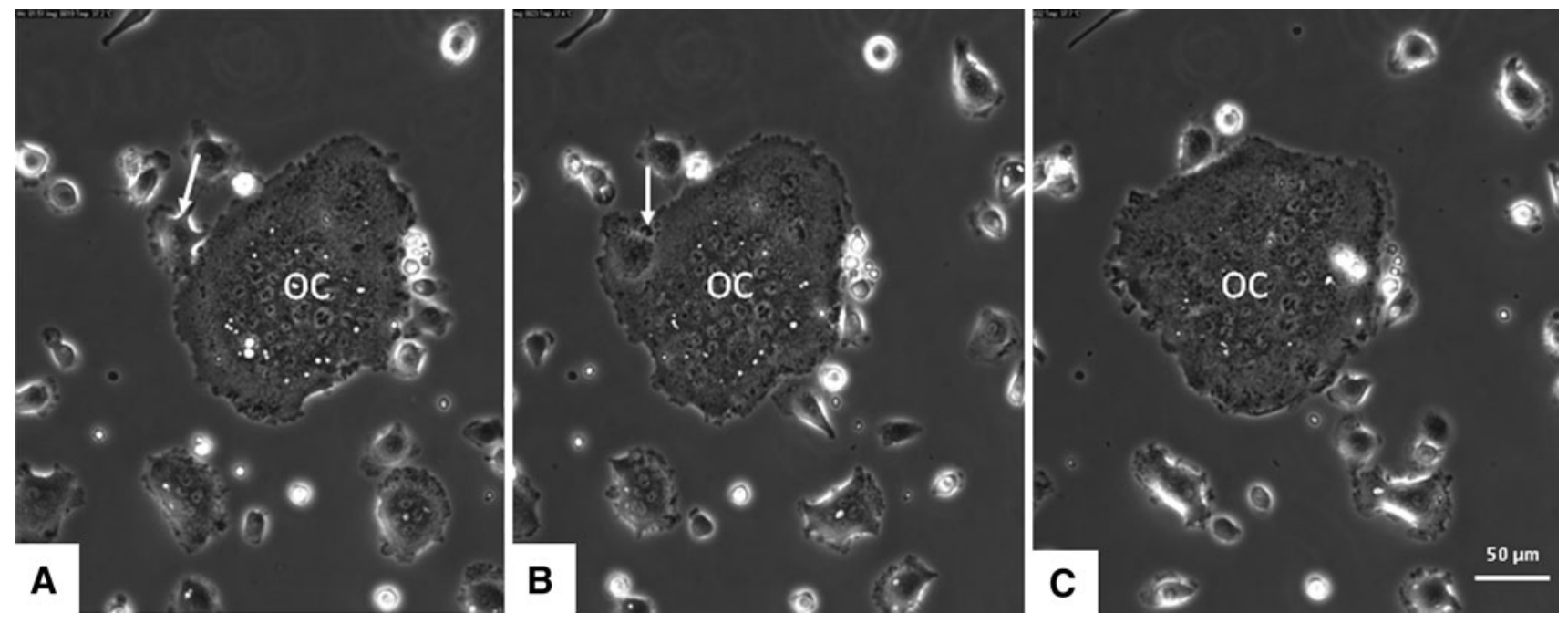

Fig. 1 Mouse bone marrow cells were precultured for 3 days in the presence of M-CSF and RANKL. Culture media were refreshed on day 3 , and cells were cultured for another $68 \mathrm{~h}$ and simultaneously followed by live cell imaging. Fusion is seen of a multinucleated cell with another multinucleated osteoclast $(O C)$. Before fusion the cells make contact with each other (arrow in $\mathbf{a}$ and b) as if to find the appropriate site to fuse. Cells are in close contact with each other (b). c Fusion has occurred 
Fig. 2 Mouse bone marrow cells (cultured in $\alpha$-MEM with M-CSF and RANKL) were followed by live cell imaging for $68 \mathrm{~h}$ after a preculture period for 3 days. In the micrograph fusion (arrow) is shown of a mononuclear cell $(m n c)$ with a multinucleated osteoclast $(O C)$
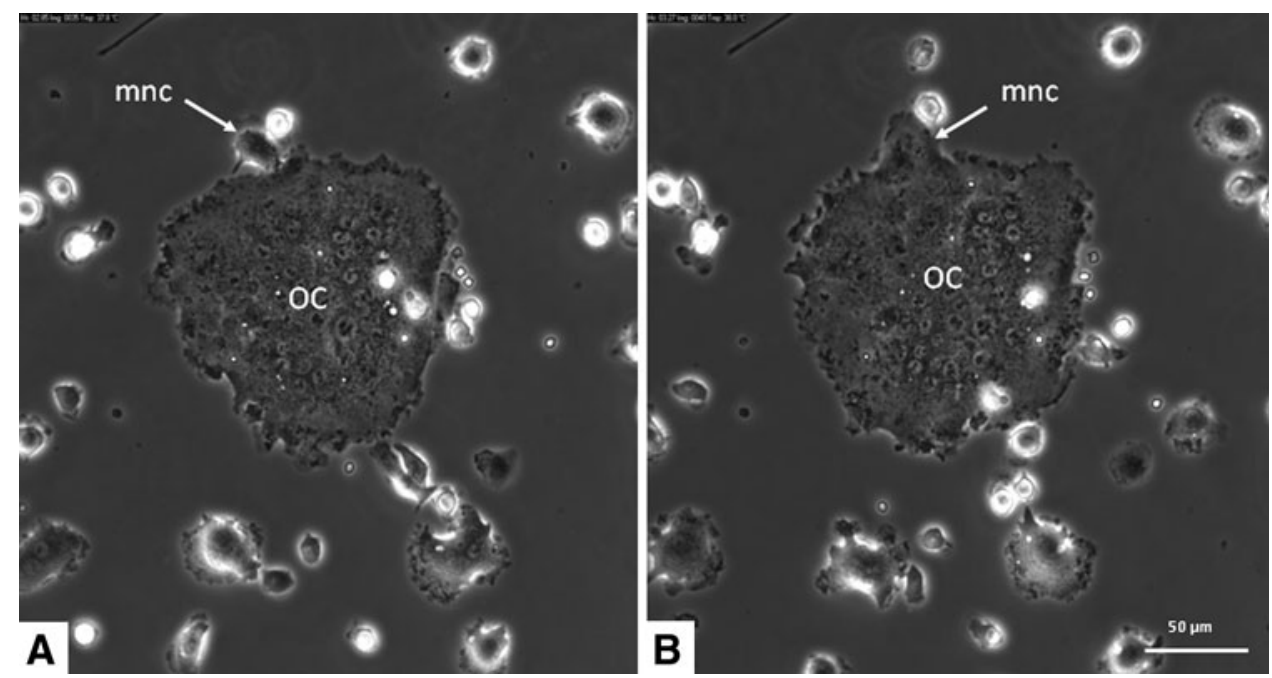

membrane of the neighboring cell (Figs. 1,2; Supplementary Data, Movie A). During most fusions, next to the fusing cells a round mononuclear cell was seen in the direct vicinity (Fig. 3).

\section{Native and In Vitro Generated Osteoclasts Can Undergo Fission}

In addition to in vitro generated osteoclasts, we used native osteoclasts isolated from rabbits. We chose the rabbit for this purpose since rabbit osteoclasts are much easier to isolate than native osteoclasts from mice.

Isolated native rabbit osteoclasts together with co-isolated osteoblast-like cells were cultured (ex vivo) and monitored for 4 days. Initially, osteoblast-like cells encircled the osteoclast, leaving a relatively small cell-free space between them and the osteoclast. The osteoclast appeared to make contact with the encircling osteoblasts by

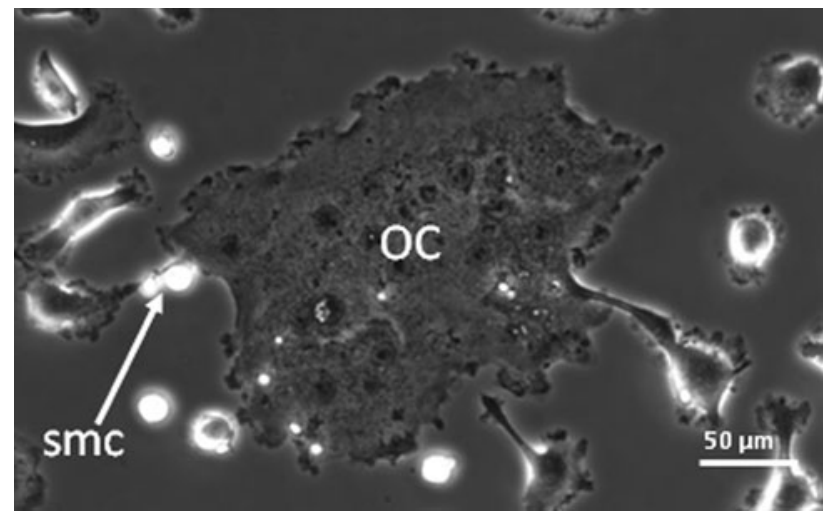

Fig. 3 Mouse bone marrow cells cultured for 6 days with M-CSF and RANKL. After refreshment of the media at day 3, cells were followed by time lapse imaging. Fusion is shown of a large osteoclast $(O C)$ with a smaller one. Note the two small mononuclear cells $(s m c)$ that are present in the direct vicinity of the site where fusion occurs cellular extensions that touched upon the surrounding cells (Supplementary Data, Movie B). During the culture period the density of osteoblast-like cells increased due to their proliferation and the cell-free area became eventually occupied by these cells.

The osteoclast moved quite extensively, and during this movement the osteoblast-like cells made space for the osteoclast. During these activities the osteoclast formed different compartments that were connected to each other with thin, tubular, cytoplasmic, bridge-like structures. Each compartment thus formed contained a number of nuclei. The thin, tubular, cytoplasmic structures bridged relatively long distances; distances up to $150 \mu \mathrm{m}$ were seen to span between the different parts of the osteoclast. These tubular structures were not firmly attached to the bottom because osteoblasts were able to move underneath them (Fig. 4c; Supplementary Data, Movie B). The different compartments were highly motile and migrated away from each other, thereby elongating the tubular connection (Fig. 4b, d). Alternatively, the compartments moved again toward each other, in the meantime shortening the tubular connecting structures. The moment the connections became very thin and long they often broke, resulting in the generation of two separate multinucleated osteoclasts (Fig. 4e).

This process of fission resulted in the generation of two or more osteoclasts, each containing a number of nuclei. The separation of the "new" cells could be either simultaneous or sequential; thus, multinucleated osteoclasts could split directly into three cells or first into two followed by another round of fission. Strikingly, we observed that the just separated cell bodies could return to each other and then fuse again.

The phenomenon of fission was also seen with mouse osteoclasts that were generated in vitro seeded on plastic or on cortical bone slices. The osteoclasts generated on plastic were followed for $68 \mathrm{~h}$ by live cell imaging (Fig. 5; 


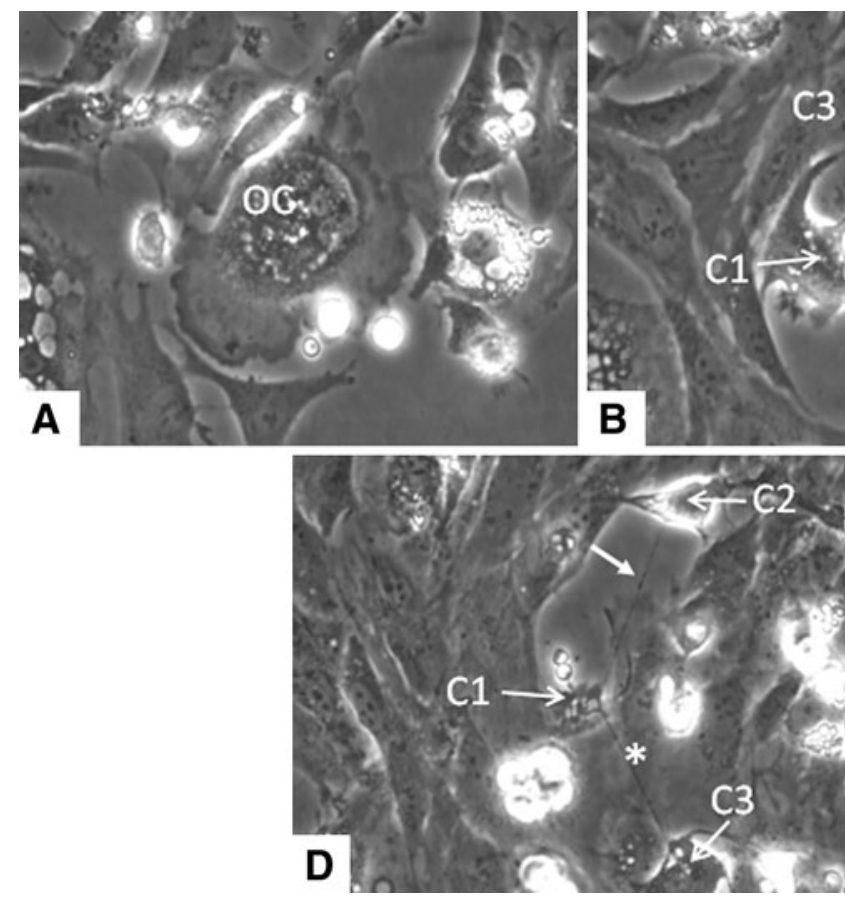

Fig. 4 In vitro generated osteoclast from mouse bone marrow. a The osteoclast $(O C)$ forms different compartments $(C 1, C 2, C 3$; shown in b-e) that are connected to each other by thin, tubular structures (closed arrow in $\mathbf{b}, \mathbf{d}$, and $\mathbf{e}$ ). Each compartment contains a number of nuclei. These tubular structures were not firmly attached to the bottom of the culture well because osteoblasts were able to move underneath
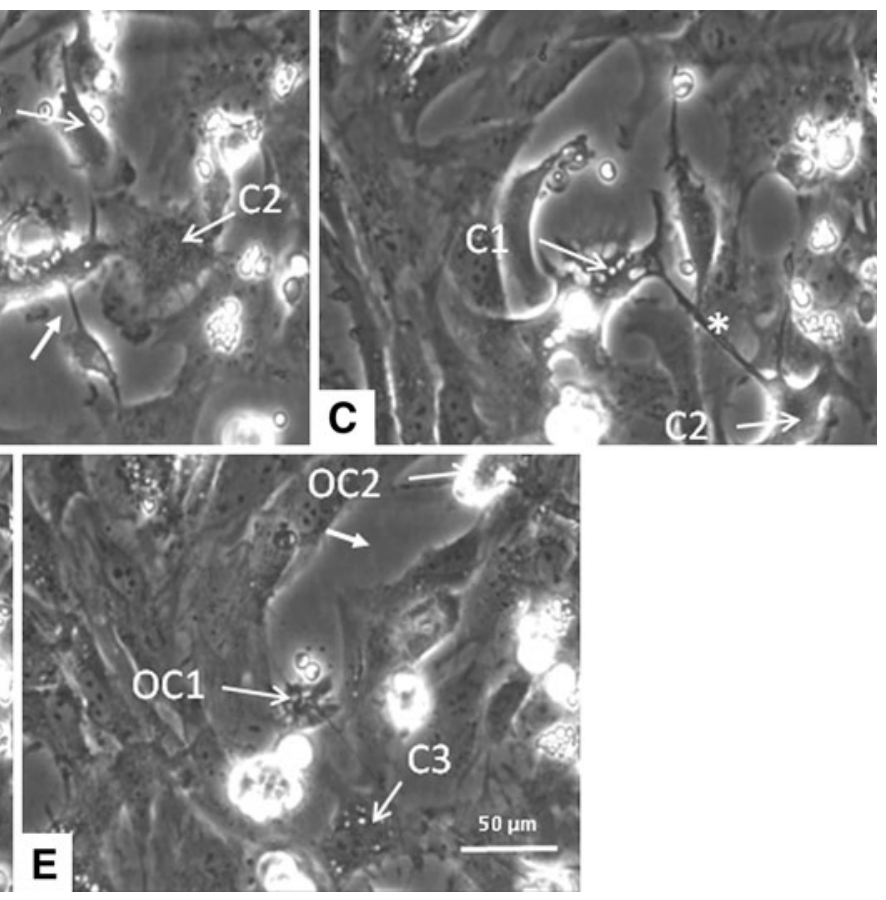

(asterisks in $\mathbf{c}$ and $\mathbf{d}$ ). Following elongation, the connections became very thin and often broke, resulting in the generation of two separate multinucleated osteoclasts (OC1, OC2) (e). Time scale of the micrographs: a was made after $13 \mathrm{~h}$ of culturing, $11 \mathrm{~h}$ later b was taken, and c-e were taken every $3 \mathrm{~h}$ thereafter

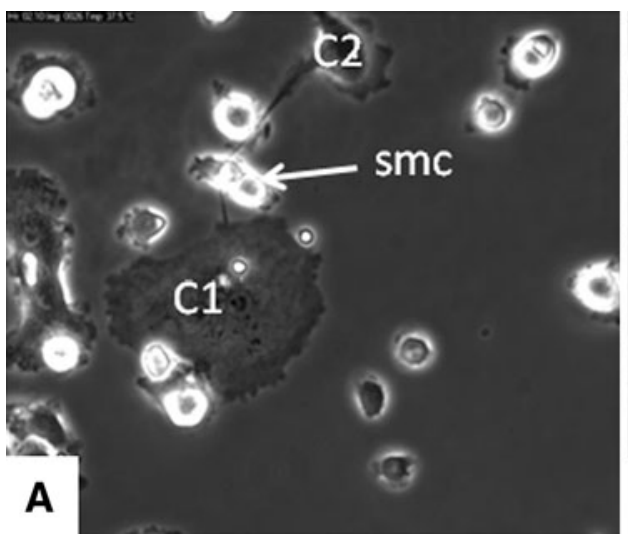

Fig. 5 Mouse bone marrow cells precultured for 3 days in the presence of M-CSF and RANKL. Culture media were refreshed on day 3 , and cells were cultured for another $68 \mathrm{~h}$ and simultaneously followed by live cell imaging. Tubular cytoplasmic structures (arrow) were formed between multinucleated compartments

Supplementary Data, Movie C). Also, here tubular cytoplasmic structures were formed between multinucleated compartments, which was followed by fission.

During the process of the breaking up of the connection, we noted an intriguing phenomenon. Small, very motile mononuclear cells moved across the bridging extension. At the site where contact between the mononuclear cell and the cytoplasmic bridge occurred, the extension was broken. This

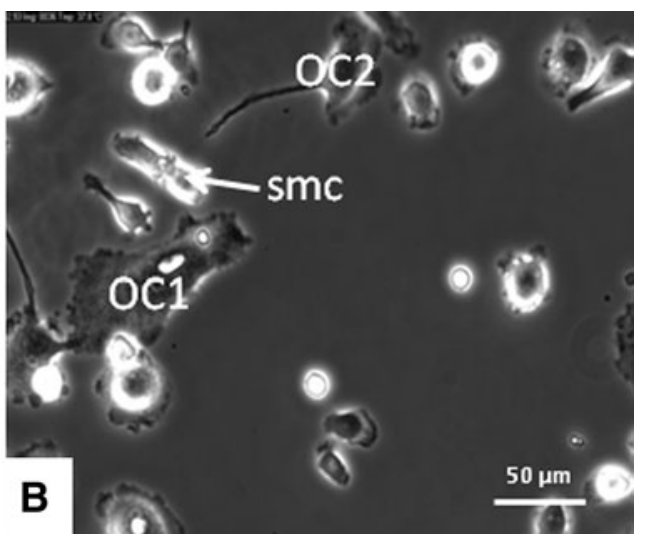

$(C 1, C 2)$. Just prior to the breaking up of the connection between compartments small mononuclear cells $(s m c)$ moved across the bridging extensions, and at the site where these cells made contact the extension was broken. Two osteoclasts $(O C 1, O C 2)$ were formed

observation strongly suggests that separation of the connection was mediated by this small mononuclear cell. Such cell-mediated separations of the connecting tubular structures occurred very frequently; it was found in $98 \%$ of the separation events (Fig. 5; Supplementary Data, Movie C). To investigate the nature of this mononuclear cell, we used a series of antibodies directed against certain subsets of mononuclear cells as well as an anti-ICAM1 antibody and 
one against MMP9. The small cells were positively labeled for ERMP20, ICAM1, and MMP9. The positive labeling of ERMP20 showed that this cell belonged to the myeloid lineage and was differentiated into a myeloid blast or monocyte [9] (Fig. 6). No positive labeling for this small mononuclear cell was found for ERMP12, Moma2, and F4/80 (not shown).

Some of the newly formed osteoclasts had the appearance of an apoptotic cell. Their shape became more round, and they partially detached from the surface; but after a while they attached again and fused with other mononuclear or multinucleated cells (Fig. 7).

The formation of compartments connected by thin extensions was also noted in cultures of osteoclasts seeded on cortical bone slices. We were not able to monitor this with live cell imaging, but frequently osteoclasts were observed consisting of different nuclei-containing compartments connected with each other by thin, cytoplasmic extensions.

To analyze whether the cells were involved in bone resorption, we visualized filamentous actin with phalloidin 488. We observed actin rings in these different osteoclast compartments (Fig. 8). Also, in some of the osteoclast compartments, we observed nuclei that were reduced in size and had an apoptotic appearance (Fig. 8).

\section{Discussion}

We visualized native mature osteoclasts and in vitro generated osteoclasts by live cell imaging and observed fusion
Fig. 6 Green fluorescent staining (Alexa-488) of the small mononuclear cell that could be involved in the separation of the osteoclast $(O C)$ compartments. Cells were labeled with anti-ERMP20 (a), anti-MMP9 (b), and antiICAM1 (c). Nuclei stained with DAPI show up in blue. Arrow indicates the labeled mononuclear cell. Asterisk marks the site where the labeled cell is in close contact with the cytoplasmic extension that connects different osteoclast parts (Color figure online)
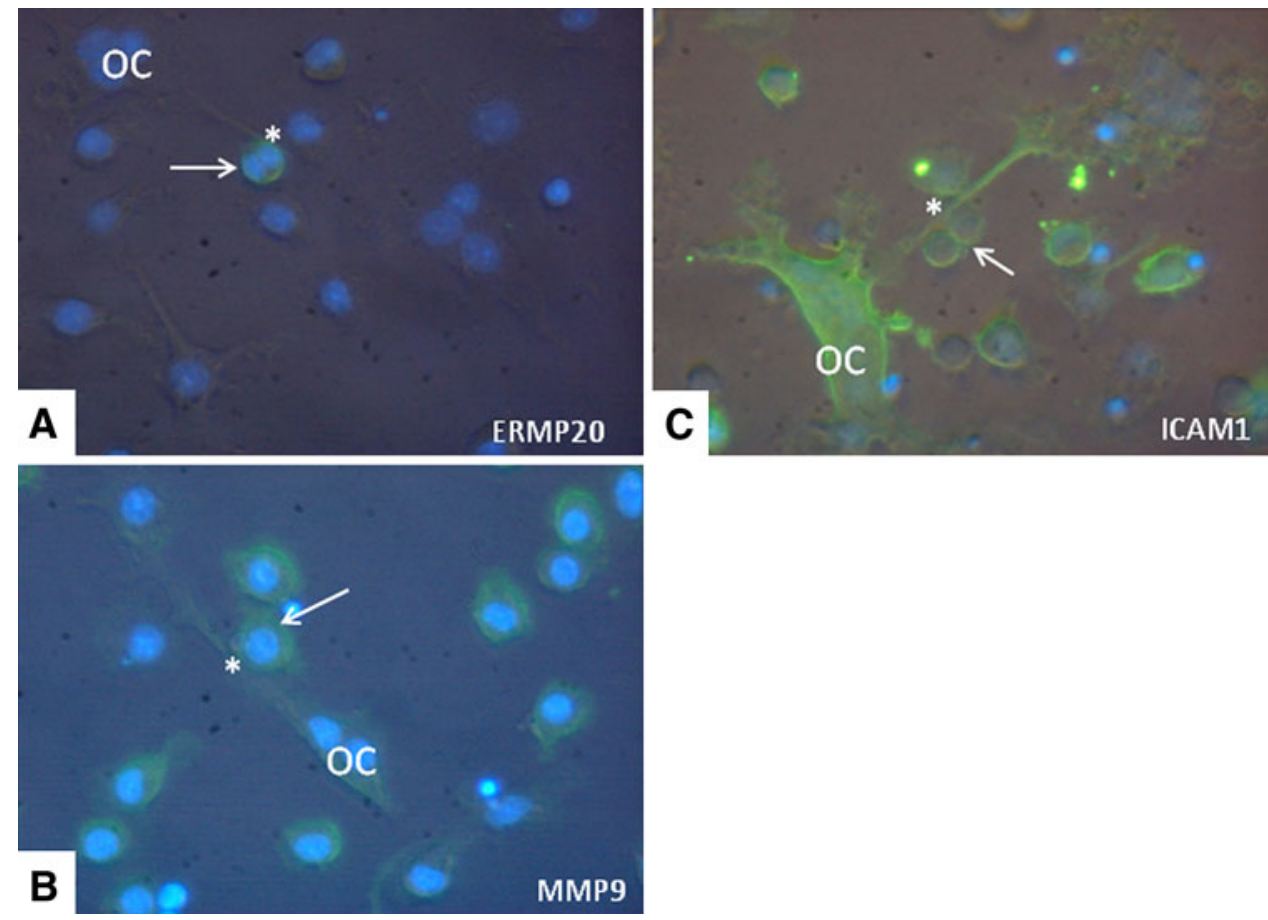
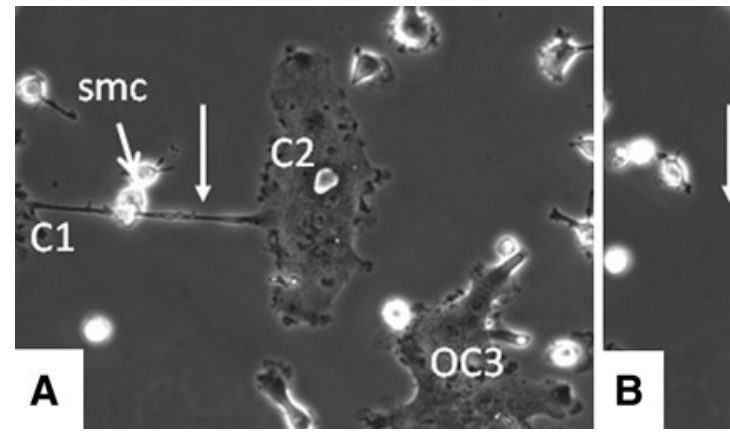

Fig. 7 Fission of an osteoclast following the formation of two compartments $(C 1, C 2)$ results in the formation of two "new" osteoclasts ( $O C 1$ and $O C 2$, shown in a and b). Subsequently, OC2 fuses with another multinucleated cell (OC3). Time span between micrographs $\mathbf{a}$ and $\mathbf{c}$ is $3 \mathrm{~h}$. The separation of the osteoclast starts $20 \mathrm{~h}$ after the start of visualization. Note the small mononuclear cells $(\mathrm{smc})$ close to the thin, tubular structure in micrograph (a) 


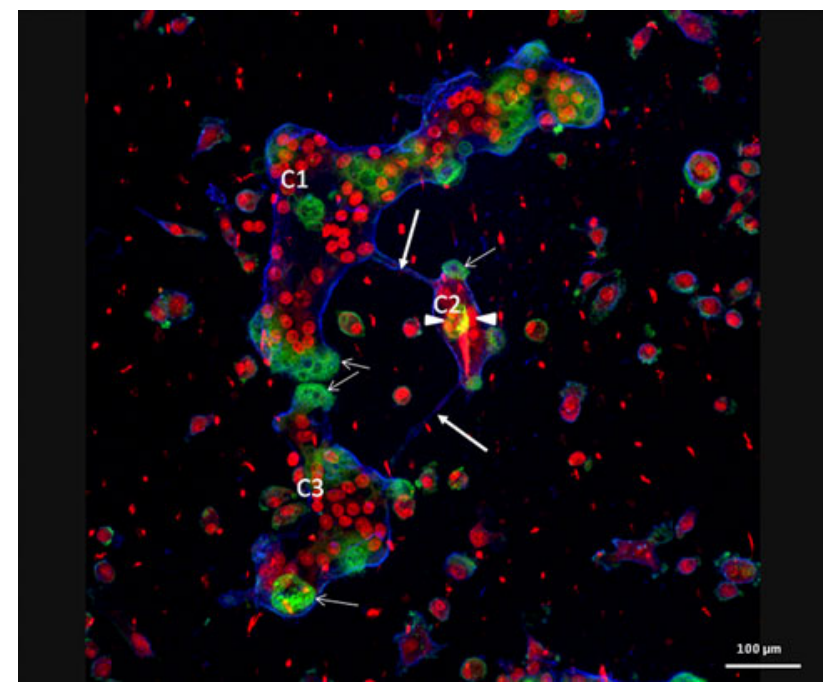

Fig. 8 The formation of compartments $(C 1, C 2, C 3)$ connected by thin, tubular structures (thick arrows) was also noted with osteoclasts seeded on cortical bone slices. Actin rings (green, thin arrows) were present in these different osteoclast compartments, indicating bone resorption activity. The osteoclast membrane is stained for CD44 (blue). Nuclei are red. In osteoclast compartment $2(C 2)$ nuclei are reduced in size and appear apoptotic (arrowheads) (Color figure online)

of all possible combinations: mononuclear with mononuclear, mononuclear with multinucleated, and multinucleated with multinucleated cells. Yet, the most exciting novel series of observations was the fission of osteoclasts. Multinucleated osteoclasts proved to have the capacity to split up in different compartments, each part containing a number of nuclei. Sometimes the nuclei of one of the newly formed parts seemed to be apoptotic, thus suggesting the ability of the cell to get rid of nonfunctional parts of the polykaryon. However, the most frequent finding was that the newly formed osteoclasts appeared to be functional given the clear presence of actin rings and their active movement.

Osteoclast fusion and fission is probably beneficial for the cell and its functional properties. The process of fusion and fission is also a common phenomenon in mitochondria. In these organelles fission and fusion was thought to play a role in apoptosis and the elimination of damaged fragments, but recently it was considered more likely that fusion and fission acts in mitochondrial quality control to form healthy and functional organelles [10]. In these organelles fusion serves to mix and unify the mitochondrial compartment, whereas fission generates new mitochondria. Fusion and fission in osteoclasts can occur for comparable reasons: to form osteoclasts with different subsets of nuclei and, therefore, with a different functionality. Recently, Youn et al. [11] reported that only a limited number of nuclei of a multinucleated osteoclast are transcriptionally active. Separation of nuclei with different expression patterns can be useful to generate osteoclasts with somewhat different functions, such as osteoclasts involved in resorption of trabecular bone and those resorbing cortical bone. In this respect it is of interest to note that Zenger and colleagues [12-14; reviewed in 15] described differences among osteoclasts associated with these different bone sites. But other functional properties of osteoclasts, such as their participation in the immune response secretion of cytokines [16, 17], interaction with osteoblasts, and recruitment of mononuclear cells from the bone marrow $[18,19]$, may lead to the presence of osteoclasts that differ in their nuclear composition.

Fusion and fission of osteoclasts resembles the phenomenon occurring with syncytiotrophoblasts in the placenta. The syncytium is a single multinucleated cell layer that covers the placenta and is in direct contact with maternal blood [20]. The syncytium regulates the exchange of nutrients and other compounds between mother and fetus. Syncytiotrophoblast cells are formed by fusion of cytotrophoblast cells. During this process the protein syncytin plays an important role [21]. It is of considerable interest to note that recently syncytin was shown to be expressed also by osteoclasts [22], thus suggesting a similarity between the fusion processes of these different cell types. During pregnancy parts of the syncytiotrophoblast are shed into the maternal blood system. These shed parts contain not only cytoplasm but also nuclei, a process comparable to the osteoclast fission noted in the present study.

Prior to fission, tubular cytoplasmic structures bridge the different compartments. The occurrence of such bridging structures was noted previously by Vesely et al. [23] and Abe et al. [24]. Yet, that these structures may form part of a rather unique property of osteoclasts, the fission of these cells, has not been described before. Zambonin and Teti [25] described the presence of cytoplasmic extensions between osteoclast parts present in medullary hen bones during hypocalcemia and suggested that osteoclasts probably shed their apoptotic nuclei. They also mentioned the presence of a mononuclear cell in close connection to the bridging extension. They suggested that this mononuclear cell either could become part of the osteoclast or was just detached from the osteoclast [25].

We visualized similar mononuclear cells in close relationship to the cellular extensions between osteoclast parts. This small mononuclear cell was found migrating over the extension just shortly before the extension broke. Given the observation that the cellular extension breaks at the site where this mononuclear cell crosses it, we propose an active participation of these cells in the process of fission and separation. Positive labeling for ERMP20 showed that this cell, comparable to osteoclast precursors, originates from the monocyte lineage. The expression of MMP9 suggests that this proteolytic enzyme plays a role in 
breaking the cytoplasmic extension. The high expression level of ICAM1 could imply that this molecule is involved in the attraction and/or binding of this cell to the cytoplasmic connection. How these cells perform such a task is unknown and needs further investigation.

Why osteoclasts show fission is not clear yet, but in line with mitochondria and syncytiotrophoblasts it is reasonable to assume that the osteoclast can regulate its own activity in this way more efficiently.

Collectively, the data presented in this study provide new insight into the dynamics of cell-cell interactions during osteoclast formation and show for the first time that mature osteoclasts can undergo fission and separate themselves into functional, smaller, yet still multinucleated cells.

Fusion and fission of osteoclasts shows that osteoclasts are very flexible cells, which have the capacity to regulate their own population in number and function, probably to adapt quickly to changing situations.

Open Access This article is distributed under the terms of the Creative Commons Attribution License which permits any use, distribution, and reproduction in any medium, provided the original author(s) and the source are credited.

\section{References}

1. Boyle WJ, Simonet WS, Lacey DL (2003) Osteoclast differentiation and activation. Nature 423:337-342

2. Perez-Amodio S, Beertsen W, Everts V (2009) (Pre-)osteoclasts induce retraction of osteoblasts before their fusion to osteoclasts. J Bone Miner Res 19:1722-1731

3. Bloemen V, de Vries TJ, Schoenmaker T, Everts V (2009) Intercellular adhesion molecule-1 clusters during osteoclastogenesis. Biochem Biophys Res Commun 385:640-645

4. Zambonin ZA, Teti A, Primavera MV (1984) Monocytes from circulating blood fuse in vitro with purified osteoclasts in primary culture. J Cell Sci 66:335-342

5. de Vries TJ, Schoenmaker T, Beertsen W, van der Neut R, Everts $\mathrm{V}$ (2005) Effect of CD44 deficiency on in vitro and in vivo osteoclast formation. J Cell Biochem 94:954-966

6. Stap J, Van Marle J, Van Veen HA, Aten JA (2000) Coating of coverslips with glow-discharged carbon promotes cell attachment and spreading probably due to carboxylic groups. Cytometry 39:295-299

7. Krawczyk PM, Stap J, Hoebe RA, van Oven CH, Kanaar R, Aten JA (2008) Analysis of the mobility of DNA double-strand breakcontaining chromosome domains in living mammalian cells. Methods Mol Biol 463:309-320

8. van Beek EM, de Vries TJ, Mulder L, Schoenmaker T, Hoeben KA, Matozaki T, Langenbach GE, Kraal G, Everts V, van den
Berg TK (2009) Inhibitory regulation of osteoclast bone resorption by signal regulatory protein alpha. FASEB J 23:4081-4090

9. de Vries TJ, Schoenmaker T, Hooibrink B, Leenen PJ, Everts V (2009) Myeloid blasts are the mouse bone marrow cells prone to differentiate into osteoclasts. J Leukoc Biol 85:919-927

10. Otera H, Mihara K (2011) Molecular mechanisms and physiologic functions of mitochondrial dynamics. J Biochem 149:241-251

11. Youn MY, Takada I, Imai Y, Yasuda H, Kato S (2010) Transcriptionally active nuclei are selective in mature multinucleated osteoclasts. Genes Cells 15:1025-1035

12. Zenger S, Hollberg K, Ljusberg J, Norgard M, Ek-Rylander B, Kiviranta R, Andersson G (2007) Proteolytic processing and polarized secretion of tartrate-resistant acid phosphatase is altered in a subpopulation of metaphyseal osteoclasts in cathepsin K-deficient mice. Bone 41:820-832

13. Zenger S, Ek-Rylander B, Andersson G (2010) Long bone osteoclasts display an augmented osteoclast phenotype compared to calvarial osteoclasts. Biochem Biophys Res Commun 394: 743-749

14. Hu Y, Ek-Rylander B, Karlstrom E, Wendel M, Andersson G (2008) Osteoclast size heterogeneity in rat long bones is associated with differences in adhesive ligand specificity. Exp Cell Res 314:638-650

15. Everts V, de Vries TJ, Helfrich MH (2009) Osteoclast heterogeneity: lessons from osteopetrosis and inflammatory conditions. Biochim Biophys Acta 1792:757-765

16. Boyce BF, Yao Z, Zhang Q, Guo R, Lu Y, Schwarz EM, Xing L (2007) New roles for osteoclasts in bone. Ann NY Acad Sci 1116:245-254

17. Boyce BF, Yao Z, Xing L (2009) Osteoclasts have multiple roles in bone in addition to bone resorption. Crit Rev Eukaryot Gene Expr 19:171-180

18. Boyce BF, Xing L (2006) Osteoclasts, no longer osteoblast slaves. Nat Med 12:1356-1358

19. Ishii M, Egen JG, Klauschen F, Meier-Schellersheim M, Saeki Y, Vacher J, Proia RL, Germain RN (2009) Sphingosine-1-phosphate mobilizes osteoclast precursors and regulates bone homeostasis. Nature 458:524-528

20. Guller S (2009) Role of the syncytium in placenta-mediated complications of preeclampsia. Thromb Res 124:389-392

21. Handwerger S (2010) New insights into the regulation of human cytotrophoblast cell differentiation. Mol Cell Endocrinol 323: 94-104

22. Soe K, Andersen TL, Hobolt-Pedersen AS, Bjerregaard B, Larsson LI, Delaisse JM (2011) Involvement of human endogenous retroviral syncytin-1 in human osteoclast fusion. Bone 48:837-846

23. Vesely P, Boyde A, Jones SJ (1992) Behaviour of osteoclasts in vitro: contact behaviour of osteoclasts with osteoblast-like cells and networking of osteoclasts for 3D orientation. J Anat 181: 277-291

24. Abe K, Ohno K, Hasegawa K (1990) Morphological relationships between osteoclasts and bone resorption surfaces on mouse parietal bones. Arch Histol Cytol 53:95-102

25. Zambonin ZA, Teti A (1981) The osteoclasts of hen medullary bone under hypocalcaemic conditions. Anat Embryol (Berl) $162: 379-392$ 